\title{
Energy Levels and Oscillator Strength of Ni XXIII
}

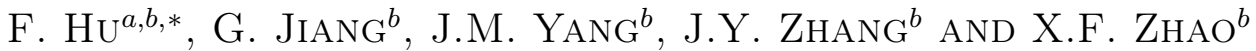 \\ ${ }^{a}$ Institute of Atomic and Molecular Physics, Sichuan University, Chengdu 610065, China \\ ${ }^{b}$ Research Center of Laser Fusion, China Academy of Engineering Physics, Mianyang 621900, China \\ (Received December 7, 2010; revised version March 3, 2011; in final form March 24, 2011)
}

Energy levels and oscillator strengths have been calculated for the fine-structure transitions among the levels of the $\left(1 s^{2}\right) 2 s^{2} 2 p^{2}, 2 s 2 p^{3}, 2 p^{4}, 2 s^{2} 2 p 3 s, 2 s^{2} 2 p 3 p$, and $2 s^{2} 2 p 3 d$ configurations of Ni XXIII using the graspVU and FAC program. The extensive configuration interaction and relativistic effects have been included while generating the wave functions. The results are compared with other recent theoretical estimates, and their accuracy is assessed.

PACS: 31.15.V-, 31.30.jc

\section{Introduction}

Emission lines of C-like ions are highly useful for diagnostics of solar, astrophysical and fusion plasmas, the interpretation of which requires the knowledge of atomic parameters such as: energy levels, transition probabilities, collision strengths and rate coefficients. Therefore, a number of researchers have reported such data over the past two decades.

Fawcett has performed elaborate calculations for a very large number of transitions, including those among the $2 s^{2} 2 p^{2}, 2 p^{4}, 2 s^{2} 2 p 3 p, 2 s 2 p^{2} 3 s$, and $2 s 2 p^{2} 3 d$ (even), and $2 s 2 p^{3}, 2 s^{2} 2 p 3 s, 2 s^{2} 2 p 3 d, 2 s 2 p^{2} 3 p, 2 p^{3} 3 s, 2 p^{3} 3 d$, and $2 s^{2} 2 p 4 s$ (odd) configurations [1]. He has used the Hartree-Fock relativistic (HFR) program, and included the configuration interaction (CI). Another calculation available in the literature, the reduced configurations of $2 s^{2} 2 p^{2}, 2 s 2 p^{3}, 2 p^{4}, 2 s^{2} 2 p 3 p, 2 s 2 p^{2} 3 s$, and $2 s^{2} 2 p 3 d$, is by Zhang and Sampson [2,3]. Also CI was included in their calculations. Aggarwal et al. have employed the fully relativistic Dirac atomic $R$-matrix code (DRAC) of Norrington and Grant for their calculations [3, 4]. The CIV3 code was also used to obtain similar results [5].

For our calculations we have used the graspVU package (a general-purpose relativistic atomic structure package), which is a modification and extension of the GRASP92 package by Parpia, Froese Fischer and Grant. To generate the wavefunctions, the extended average level (EAL) was our option, in which a weighted (proportional to $2 J+1)$ trace of Hamiltonian matrix is minimized. This produces a compromise set of orbitals describing closely states with moderate accuracy. Also, as in the calculations by other workers, we have included CI among the above basic six configurations. Further relativistic corrections arising from the Breit interaction and QED effects have also been included. Furthermore, in order to assess the accuracy of our results we have performed parallel calculations using the flexible atomic code (FAC) of

\footnotetext{
* corresponding author; e-mail: hufengscu@139.com
}

$\mathrm{Gu}[6]$. This is also a fully relativistic code which provides a variety of atomic parameters, and yields results comparable to grasp, as already shown for three Mg-like ions by Aggarwal et al. [7]. Thus results from FAC will be helpful in assessing the accuracy of our energy levels and radiative rates.

As is well known, nickel is an important impurity element in fusion reactors, and many emission lines of its ions have been observed in solar plasmas. So in our previous paper, we have reported the results between Ni XIX and Ni XXVII [8]. Though we have obtained some for $\mathrm{Ni}$ XXIII (only $k_{\alpha}$ and $K_{\beta}$ transitions), systematic calculations on carbon-like nickel are still fragmentary. It is essential to study the properties of these lines in detail, so our investigation is motivated by the need for accurate transition data in a variety of scientific applications.

\section{Methods}

The EAL version of the multi-configuration DiracFock (MCDF) method is used to calculate the transitions. The theoretical basis of our present computational approach has been widely discussed elsewhere [9-12]. Hence we only repeat the essential features here.

The GRASPVU Package is based on the MCDF method. In the MCDF method the atom is represented by atomic state functions (ASF), which are a linear combination of configuration state functions (CSF)

$$
\Psi(\gamma J)=\sum_{i} c_{i} \Phi_{i}\left(\alpha_{i} J\right),
$$

where the CSF $\Phi(\alpha J)$ are antisymmetrized linear combinations of relativistic orbital products of the form

$$
\Phi(\boldsymbol{r})=\frac{1}{r}\left(\begin{array}{ll}
P_{n k}(\boldsymbol{r}) & \chi_{k m}(\boldsymbol{r}) \\
\mathrm{i} Q_{n k}(\boldsymbol{r}) & \chi_{-k m}(\boldsymbol{r})
\end{array}\right) .
$$

Here $k$ is the relativistic angular momentum, $P_{n k}(\boldsymbol{r})$ and $Q_{n k}(\boldsymbol{r})$ are the large and small component radial wave functions and $\chi_{k m}(\boldsymbol{r})$ is the spinor spherical harmonic in the $L S J$ coupling scheme. The CSF are constructed from one-electron orbitals, according to the well-known rules of symmetry (parity and angular momenta)(Grant 
et al. 1980). The goal is a systematic approach, where all important contributions are included. This allows for monitoring the convergence of different properties and plausible estimates of uncertainties. The foundation of the method is an active set of orbitals, which is used to generate all possible CSF. The size, and thereby the accuracy, of a certain approach is therefore defined by the size of the active set of orbitals. It is thus straightforward to define a systematic approach, by just increasing the orbital set in a systematic fashion.

The Dirac-Coulomb Hamiltonian can be written as

$$
\widehat{H}^{\mathrm{DC}}=\sum_{i}^{N} \widehat{H_{\mathrm{D}}}(i)+\sum_{i=1}^{N-1} \sum_{j=i+1}^{N}\left|\widehat{r_{i}}-\widehat{r_{j}}\right|^{-1} \text {. }
$$

All the dominant interactions in an $N$-electron atom or ion are included in the Dirac-Coulomb Hamiltonian. In Eq. (3), the first term is the one-body contribution for an electron due to the kinetic energy and interaction with the nucleus. The two-body Coulomb interaction between the electrons comprises the second term. Higher order modifications to Eq. (3) due to the transverse electromagnetic interaction and the radiative corrections are treated via perturbation theory.

This work was performed using an expansion of configuration interaction based on the MCDF method. To build a CSF expansion, the restrictive active space methods were used. The idea of the active space methods is to consider only electrons from the active set and to excite them from the occupied orbitals to unoccupied ones. The orbital was increased systematically in order to monitor the convergence of the calculation. Since the orbitals with the same principal quantum number $n$ often have similar energies, the active set is usually enlarged in steps of orbital layers. It is convenient to refer to the $\{1 s, 2 s, 2 p, 3 s, 3 p, 3 d\}$ set of orbitals as the $n=3$ orbital layer, $\{1 s, 2 s, 2 p, \ldots 4 s, 4 p, 4 d, 4 f\}$ as $n=4$, etc. Larger orbital sets can result in a considerable increase of computational time required for the problem, and appropriate restrictions may be necessary. Based on above calculation method, we considered the active space consisting of all the orbitals in the set with principal quantum number $n=1-5$ and $l=s$ to $f$. All electrons were treated as reference configurations throughout the present calculations.

Succeeding the generation of the CSF sets, we perform angular integrations for the matrix elements of the many-electron Dirac-Coulomb Hamiltonian. To generate initial estimates for radial orbitals, we used a ThomasFermi potential. The optimizations were carried out layer by layer to obtain the orbitals requested in next CI calculation. In this scheme, first we optimized the orbitals with $n \leq 3$ in the reference CSFs. Then we optimized the orbitals for $n=4$ layer and keep the $n \leq 3$ orbitals fixed. With this scheme we optimized $n=6$. The optimization is on the $(2 J+1)$ weighted average of all fine structure levels belonging to an $L S$ term and the driven to convergence with the self-consistency criteria set to $10^{-8}$. In the EAL calculation where states of different angular momentum $J$ and parity are obtained simultaneously, we use the block structure of the Hamiltonian matrix. Since both $J$ and parity are good quantum numbers and the basis functions are already eigenstates of these quantities, it is preferable to re-arrange the order of the basis functions so that all the states with the same $J$ and parity are grouped into a particular block.

After obtaining the set of radial functions, relativistic configuration interaction calculations were carried out to determine CSF expansion coefficients by diagonalizing the Hamiltonian matrix that included the frequency-dependent Breit interaction, vacuum polarization and self-energy correction. In this implementation of the RCI program an iterative Davidson method was used together with a spare matrix representation allowing for large expansions.

Finally, the mixing coefficient was transformed from the block format to non-block format, and then the initial and final state orbital sets were transformed to become biorthogonal. Then we can compute oscillator strengths, line strength, transition probability between biorthogonal states and each matrix element can be evaluated by the standard Racah algebra.

\section{Results and discussions}

\subsection{Energy levels}

As mentioned above, we have included in the CI expansion all possible angular momentum couplings of the $\left(1 s^{2}\right) 2 s^{2} 2 p^{2}, 2 s^{2} 2 p n l, 2 s 2 p^{2} n l, 2 p^{3} n l, 2 p^{4}$ and $2 s 2 p n l n^{\prime} l^{\prime}$. In order to compare our results between NIST (http://physics.nist.gov/PhysRefData), FAC and multi-configuration Hartree-Fock (MCHF), we will focus our results on the lowest 46 levels. These levels include the levels of $\left(1 s^{2}\right) 2 s^{2} 2 p^{2}, 2 s 2 p^{3}, 2 p^{4}, 2 s^{2} 2 p 3 s, 2 s^{2} 2 p 3 p$ and $2 s^{2} 2 p 3 d$.

We list in Table I our calculated energies from graspVU and FAC. Also included in this table are the experimental energies compiled by NIST, those obtained by Froese Fischer who adopted MCHF code [13] and the energies calculated by the method of multi-configuration DiracHartree-Fock (MCDHF) method. The first column of each table provides an index for the corresponding level.

The graspVU results obtained with the inclusion of Breit and QED corrections are listed in Table I. In order to become closer to NIST energy, the results without the correction of Breit and QED were not taken into account. This is because the effect of the inclusion of Breit and QED corrections on the energy levels is limited to $<0.01 \%$. From Table I, we can see that our results are in a good agreement with NIST. It may be noted that our results from graspVU are higher up to $2.08 \%$ - see level 6. The calculation did by FAC differ from the NIST compilation by up to $3.1 \%$. So the energy of level 6 should be checked for another theory or a new experiment. This is because the present calculated energy differences are less than $1 \%$. This situation of energy 
order is frequently encountered in all theoretical calculations, especially when the energy separation of the levels is very small. Furthermore, in the absence of definitive experimental level energies in most of the cases, a correct energy order cannot be confirmed. In any event, it does not affect the computed values of the oscillator strengths.

The energy levels of Tachiev from the MCHF code are, in general, comparable in both magnitude (within 9\%) and orderings with those of NIST and our calculations from graspVU. It may also be noted that like our calculations from graspVU, no adjustment of Hamiltonian matrices have been made by them. Their energy level of $1 s^{2} 2 s^{2} p^{2}{ }^{1} S_{0}$ is anomalous, as it is up to $9.6 \%$ higher than the experimental compared to $3 \%$ for graspVU and $2 \%$ for FAC, although the difference is only $62550 \mathrm{~cm}^{-1}$. From Table I, we can include that the MCHF results are a little higher than the experimental results from NIST. From Table I, we can see that the new MCDHF results are in a good agreement with the NIST results, the biggest difference is only $0.31 \%$. Our graspVU and FAC results agree well with the new MCDHF results (within 1\%).

In our calculations from FAC we have included all possible combinations of the $n=2$ and $n=3$ configurations, in addition to $1 s^{2} 2 s^{2} 2 p 4 l, 1 s^{2} 2 s^{2} 2 p 5 l, 1 s^{2} 2 s^{2} 3 l 4 l$, $1 s^{2} 2 s^{2} 3 l 5 l$ and inner excitation. The results obtained from this calculation are listed in Table I. Differences between the two sets of calculation from graspVU and FAC are within $1 \%$ for a majority of levels, except for $1 s^{2} 2 s 2 p^{3}$ ${ }^{1} P_{1}$ which is up to $2 \%$. But there are no major differences between our calculations from graspVU and FAC, in both magnitude and ordering. Based on these comparisons, we may confidently state that the present calculations from graspVU and FAC are probably accurate.

\subsection{Radiative rates}

The absorption oscillator strengths $\left(f_{i j}\right)$ and radiative rate $A_{j i}\left(\right.$ in $\left.^{-1}\right)$ for a transition $i \rightarrow j$ are related by the following expression:

$$
f_{i j}=\frac{m c}{8 \pi^{2} e^{2}} \lambda_{i j}^{2}=1.49 \times 10^{-6} \lambda_{i j}^{2}\left(\omega_{j} / \omega_{i}\right) A_{j i},
$$

where $m$ and $e$ are the electron mass and charge, respectively, $c$ is the velocity of light, $\lambda_{j i}$ is the transition energy/wavelength in $\AA$, and $\omega_{i}$ and $\omega_{j}$ are the statistical weights of the lower $i$ and upper $j$ levels, respectively. Similarly, the oscillator strength $f_{i j}$ (dimensionless) and the line strength $S$ (in atomic unit, 1 a.u. $\left.=6.460 \times 10^{-36} \mathrm{~cm}^{2} \mathrm{esu}^{2}\right)$ are related by the following standard equations:

For the electric dipole (E1) transitions:

$$
A_{j i}=\frac{2.0261 \times 10^{18}}{\omega_{j} \lambda_{j i}^{3}} S \text { and } f_{i j}=\frac{303.75}{\lambda_{j i} \omega_{i}} S .
$$

For the magnetic dipole (M1) transitions:

$$
A_{j i}=\frac{2.0694 \times 10^{13}}{\omega_{j} \lambda_{j i}^{3}} S \text { and } \quad f_{i j}=\frac{4.0144 \times 10^{-3}}{\lambda_{j i} \omega_{i}} S .
$$

$$
A_{j i}=\frac{1.1199 \times 10^{18}}{\omega_{j} \lambda_{j i}^{5}} S \text { and } f_{i j}=\frac{167.89}{\lambda_{j i}^{3} \omega_{i}} S .
$$

For the magnetic quadrupole (M2) transitions:

$$
A_{j i}=\frac{1.4910 \times 10^{13}}{\omega_{j} \lambda_{j i}^{5}} S \text { and } f_{i j}=\frac{2.236 \times 10^{-3}}{\lambda_{j i}^{3} \omega_{i}} S \text {. }
$$

In Table II, we present $\mathrm{E} 1$ radiative rates $\left(A_{j i}\right.$ in $\left.\mathrm{s}^{-1}\right)$ and oscillator strengths $f_{i j}$, in length form only, for the transitions among the 46 levels of Ni XXVII, and those radiative rates $\leq 10^{-6} \mathrm{~s}^{-1}$ were not listed in Table II. All the oscillator strengths and radiative rates were calculated by the graspVU code. The indices used to represent the lower and upper levels of a transition have already been defined in Table I. Here the results are obtained with the Breit and QED correction. It is clear that our results are in a general agreement with the MCHF and MCDHF results. Not all the E2, M1 and M2 results were listed here. We only gave some forbidden transitions compared with other results available in the literature.

The transitions probabilities obtained by several authors are shown in Table III. Nussbaumer and Rusca (NR) [14] and Czyzak and Poirer (CP) [15] used the experimental levels for calculating the transitions probabilities, whereas Cheng et al. (CDK) [16] use the theoretically calculated energy level value. Since CKD used theoretically calculated energy levels, CP repeated their calculations using CKD's energy level values in the determination of transition probabilities. The results of these calculations are shown in the column $\mathrm{CP}^{*}[15]$. Also our results from graspVU, results from MCHF and NIST are listed in Table III. Here Am and Aq are short for magnetic dipole and electric quadrupole transition probabilities.

In general, for the forbidden transitions involving the ${ }^{3} P,{ }^{1} D$ and ${ }^{1} S$ terms, the similarities and differences can be summarized in the following way:

(1) There is a general agreement between the values of NR, CKD, graspVU, MCHF and NIST. The results are no major differences in both magnitude and ordering.

(2) For ${ }^{3} P_{1}-{ }^{3} P$ electric quadrupole transition probabilities, the difference for $\mathrm{CP}$ and $\mathrm{CP}^{*}$ are significant and sizeable. These must be attributed to the primary cause for the difference in the values obtained.

(3) The ratio of $\mathrm{NR}, \mathrm{CP}, \mathrm{CP}^{*}$, graspVU and $\mathrm{MCHF}$ compared to $\mathrm{NR}$ are listed in Fig. 1. It is obvious that the $\mathrm{CP}$ and $\mathrm{CP}^{*}$ badly agree with others.

(4) Our transition probabilities (obtained by including the relativistic effects) are in excellent agreement (within $7 \%$ ) with the experimental values. The good agreement appears in the electric quadrupole transition probabilities for ${ }^{3} P_{2}-{ }^{1} D_{2}$. The difference is smaller than $0.001 \%$.

For the electric quadrupole (E2) transitions: 


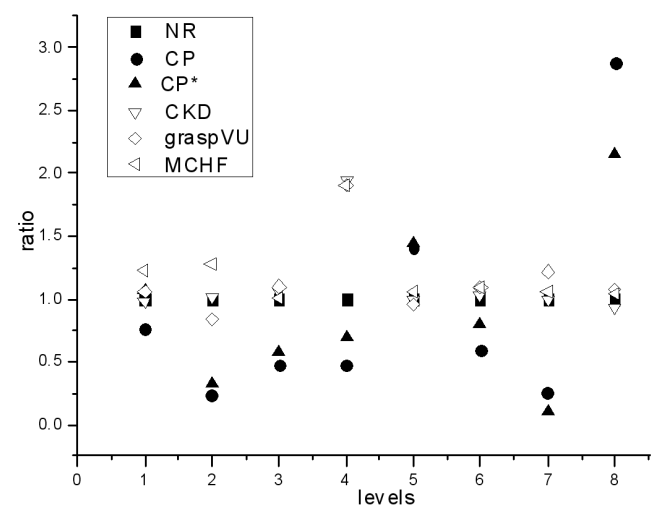

Fig. 1. The ratios of $\mathrm{NR}, \mathrm{CP}, \mathrm{CP}^{*}$, graspVU and MCHF compared to NR.

Finally, in analyses of these data it appears that the results obtained will depend on whether included in the wave function calculations, are the corrections effects including those outside the complex which are significant for the first several ions, the number of configurations considered, the relativistic results, and the energy level values used. Though our results are in a good agreement with others, they still needed to be improved by obtaining the experimental energy level values.

\section{Conclusions}

In this paper we have reported the oscillator strengths as well as the radiative rates for transitions among the $\left(1 s^{2}\right) 2 s^{2} 2 p^{2}, 2 s 2 p^{3}, 2 p^{4}, 2 s^{2} 2 p 3 s, 2 s^{2} 2 p 3 p$, and $2 s^{2} 2 p 3 d$ configurations of C-like Ni XXIII. Relativistic effects as well as elaborated CI have been included in the calculations. The results have been compared to NIST and MCHF. The energy levels are calculated by MCDF and FAC. Compared to NIST, the biggest differences is only $2.08 \%$. The forbidden transitions have been calculated by several authors and have been compared and discussed in detail in the literature. Our results are general in a good agreement with other results.

\section{Acknowledgments}

This work was supported by National Natural Science Fund (10874156) and the key item of Science and Technology Foundation of CAEP (2010A0102003).

\section{References}

[1] B.C. Fawcett, At. Data Nucl. Data Tables 37, 367 (1987).

[2] H.L. Zhang, D.H. Sampson, At. Data Nucl. Data Tables 63, 275 (1996).

[3] H.L. Zhang, D.H. Sampson, At. Data Nucl. Data Tables 65, 183 (1997).

[4] K.M. Aggarwal, F.P. Keenan, J. Phys B 32, 3585 (1999).

[5] K.M. Aggarwal, F.P. Keenan, A.Z. Msezane, ApJS 136, 763 (2001).

[6] M.F. Gu, ApJ 582, 1241 (2003).

[7] K.M. Aggarwal, V. Tayal, G.P. Gupta, F.P. Keenan, At. Data Nucl. Data Tables 93, 615 (2007).

[8] F. Hu, G. Jiang, W. Hong, L.H. Hao, Eur. Phys. J. D 49, 293 (2008).

[9] K.G. Dyall, I.P. Grant, C.T. Johnson, F.A. Parpia, E.P. Plummer, Comput. Phys. Commun. 55, 425 (1989).

[10] F.A. Parpia, C. Froese Fischer, I.P. Grant, Comput. Phys. Commun. 94, 249 (1996).

[11] A. Stathopoulos, C. Froese Fisher, Comput. Phys. Commun. 79, 1 (1994).

[12] F. Hu, G. Jiang, J.M. Yang, C.K. Wang, X.F. Zhao, L.H. Hao, Eur. Phys. J. D 61, 15 (2011).

[13] C.F. Fischer, MCHF Collection: http://atoms. vuse.vanderbilt.edu/ .

[14] H. Nussbaumer, C. Rusca, A\&A 64, 139 (1979).

[15] Czyzak, Poirer, Astrophys. Space Sci. 116, 21 (1985).

[16] K.T. Cheng, Y.K. Kim, J.P. Desclaux, At. Data Nucl. Data Tables 24, 111 (1979).

Selected forbidden transition probabilities of the Ni XXIII.

TABLE III

\begin{tabular}{c|c|c|c|c|c|c|c|c}
\hline \hline Transition & Type & NR & CP & CP* & CKD & graspVU & MCHF & NIST \\
\hline${ }^{3} P_{0}-{ }^{3} P_{1}$ & $\mathrm{Am}$ & $2.11(+4)$ & $1.61(+4)$ & $2.25(+4)$ & $2.08(+4)$ & $2.23(+4)$ & $2.59(+4)$ & $2.07(+4)$ \\
& $\mathrm{Aq}$ & $2.93(+0)$ & $0.72(+0)$ & $0.97(+0)$ & $2.99(+0)$ & $2.46(+0)$ & $3.74(+0)$ & \\
${ }^{3} P_{1}-{ }^{3} P_{2}$ & $\mathrm{Am}$ & $1.29(+3)$ & $0.61(+3)$ & $0.74(+3)$ & $1.38(+3)$ & $1.42(+3)$ & $1.31(+3)$ & $1.32(+3)$ \\
& $\mathrm{Aq}$ & & $4.39(-3)$ & $5.20(-3)$ & $1.23(-2)$ & $1.52(-2)$ & $1.20(-2)$ & \\
${ }^{3} P_{0}-{ }^{1} D_{2}$ & $\mathrm{Aq}$ & $2.16(-1)$ & $1.03(-1)$ & $1.50(-1)$ & $4.21(-1)$ & $4.12(-1)$ & $4.11(-1)$ & \\
${ }^{3} P_{1}-{ }^{1} D_{2}$ & $\mathrm{Am}$ & $4.22(+4)$ & $5.92(+4)$ & $6.10(+4)$ & $4.23(+4)$ & $4.03(+4)$ & $4.44(+4)$ & $4.1(+4)$ \\
& $\mathrm{Aq}$ & & $6.82(+0)$ & $7.52(+0)$ & $6.09(+0)$ & $6.37(+0)$ & $6.58(+0)$ & \\
\hline${ }^{3} P_{2}-{ }^{1} D_{2}$ & $\mathrm{Am}$ & $3.63(+4)$ & $2.18(+4)$ & $2.91(+4)$ & $3.75(+4)$ & $3.95(+4)$ & $3.97(+4)$ & $3.7(+4)$ \\
& $\mathrm{Aq}$ & & $3.24(+0)$ & $4.78(+0)$ & $7.35(+0)$ & $7.31(+0)$ & $7.90(+0)$ & $7.30(+0)$ \\
${ }^{3} P_{1}-{ }^{1} S_{0}$ & $\mathrm{Am}$ & $2.94(+5)$ & $0.77(+5)$ & $0.33(+5)$ & $2.95(+5)$ & $3.58(+5)$ & $3.09(+5)$ & \\
${ }^{3} P_{2}-{ }^{1} S_{0}$ & $\mathrm{Aq}$ & $1.38(+2)$ & $3.96(+2)$ & $2.97(+2)$ & $1.28(+2)$ & $1.48(+2)$ & $1.44(+2)$ &
\end{tabular}


Levels of Ni XXIII and their threshold energies (in $\mathrm{cm}^{-1}$ ).

TABLE I

\begin{tabular}{|c|c|c|c|c|c|c|c|}
\hline & Configuration & Level & Expt. & graspVU & FAC & $\mathrm{MCHF}^{a}$ & $\mathrm{MCDHF}^{b}$ \\
\hline 1 & $1 s^{2} 2 s^{2} 2 p^{2}$ & ${ }^{3} P_{0}$ & 0.0 & 0.0 & 0.0 & 0 & 0.0 \\
\hline 2 & $1 s^{2} 2 s^{2} 2 p^{2}$ & ${ }^{3} P_{1}$ & 109770 & 109660 & 109650 & 118242 & 109575 \\
\hline 3 & $1 s^{2} 2 s^{2} 2 p^{2}$ & ${ }^{3} P_{2}$ & 161922 & 161116 & 162598 & 171355 & 161957 \\
\hline 4 & $1 s^{2} 2 s^{2} 2 p^{2}$ & ${ }^{1} D_{2}$ & 324640 & 323956 & 326095 & 337300 & 324881 \\
\hline 5 & $1 s^{2} 2 s^{2} 2 p^{2}$ & ${ }^{1} S_{0}$ & 463900 & 463842 & 462016 & 476227 & 465393 \\
\hline 6 & $1 s^{2} 2 s 2 p^{3}$ & ${ }^{5} S_{2}$ & 586890 & 574646 & 575056 & 649996 & 585792 \\
\hline 7 & $1 s^{2} 2 s 2 p^{3}$ & ${ }^{3} D_{1}$ & 894100 & 893694 & 896642 & 958215 & 895170 \\
\hline 8 & $1 s^{2} 2 s 2 p^{3}$ & ${ }^{3} D_{2}$ & 900000 & 900862 & 901218 & 965743 & 900693 \\
\hline 9 & $1 s^{2} 2 s 2 p^{3}$ & ${ }^{3} D_{3}$ & 941400 & 943718 & 942292 & 1010834 & 941921 \\
\hline 10 & $1 s^{2} 2 s 2 p^{3}$ & ${ }^{3} P_{0}$ & 1064900 & 1065497 & 1066570 & 1131249 & 1065736 \\
\hline 11 & $1 s^{2} 2 s 2 p^{3}$ & ${ }^{3} P_{1}$ & 1078350 & 1078446 & 1080847 & 1145460 & 1079499 \\
\hline 12 & $1 s^{2} 2 s 2 p^{3}$ & ${ }^{3} P_{2}$ & 1104750 & 1105702 & 1107326 & 1173443 & 1105906 \\
\hline 13 & $1 s^{2} 2 s 2 p^{3}$ & ${ }^{3} S_{1}$ & 1250470 & 1258144 & 1263911 & 1315641 & 1253646 \\
\hline 14 & $1 s^{2} 2 s 2 p^{3}$ & ${ }^{1} D_{2}$ & 1304640 & 1306546 & 1317418 & 1375480 & 1307885 \\
\hline 15 & $1 s^{2} 2 s 2 p^{3}$ & ${ }^{1} P_{1}$ & 1459700 & 1453833 & 1474529 & 1531593 & 1464350 \\
\hline 16 & $1 s^{2} 2 p^{4}$ & ${ }^{3} P_{2}$ & 1864700 & 1877375 & 1879943 & 1965209 & 1867113 \\
\hline 17 & $1 s^{2} 2 p^{4}$ & ${ }^{3} P_{0}$ & 1977400 & 1981320 & 1993795 & 2104904 & 1979779 \\
\hline 18 & $1 s^{2} 2 p^{4}$ & ${ }^{3} P_{1}$ & 1999400 & 1998597 & 2012835 & 2075868 & 2001281 \\
\hline 19 & $1 s^{2} 2 p^{4}$ & ${ }^{1} D_{2}$ & 2080600 & 2094973 & 2102172 & 2187174 & 2083559 \\
\hline 20 & $1 s^{2} 2 p^{4}$ & ${ }^{1} S_{0}$ & 2348200 & 2350139 & 2373772 & 2454396 & 2352922 \\
\hline 21 & $1 s^{2} 2 s^{2} 2 p 3 s$ & ${ }^{3} P_{0}$ & & 9140361 & 9347392 & 9219471 & 9114110 \\
\hline 22 & $1 s^{2} 2 s^{2} 2 p 3 s$ & ${ }^{3} P_{1}$ & & 9149645 & 9156471 & 9378129 & 9124064 \\
\hline 23 & $1 s^{2} 2 s^{2} 2 p 3 s$ & ${ }^{3} P_{2}$ & & 9300863 & 9166193 & 9403050 & 9276328 \\
\hline 24 & $1 s^{2} 2 s^{2} 2 p 3 s$ & ${ }^{1} P_{1}$ & & 9302388 & 9321823 & 9209682 & 9302059 \\
\hline 25 & $1 s^{2} 2 s^{2} 2 p 3 p$ & ${ }^{1} P_{1}$ & & 9544666 & 9352483 & 9504349 & 9383882 \\
\hline 26 & $1 s^{2} 2 s^{2} 2 p 3 p$ & ${ }^{3} D_{1}$ & & 9321153 & 9430968 & 9430243 & 9311541 \\
\hline 27 & $1 s^{2} 2 s^{2} 2 p 3 p$ & ${ }^{3} D_{2}$ & & 9403775 & 9431725 & 9510066 & 9389169 \\
\hline 28 & $1 s^{2} 2 s^{2} 2 p 3 p$ & ${ }^{3} D_{3}$ & & 9535152 & 9450929 & 9648138 & 9521994 \\
\hline 29 & $1 s^{2} 2 s^{2} 2 p 3 p$ & ${ }^{3} S_{1}$ & & 9512437 & 9557945 & 9655073 & 9529934 \\
\hline 30 & $1 s^{2} 2 s^{2} 2 p 3 p$ & ${ }^{3} P_{0}$ & & 9404971 & 9566197 & 9514379 & 9395464 \\
\hline 31 & $1 s^{2} 2 s^{2} 2 p 3 p$ & ${ }^{3} P_{1}$ & & 9397037 & 9578553 & 9660297 & 9502751 \\
\hline 32 & $1 s^{2} 2 s^{2} 2 p 3 p$ & ${ }^{3} P_{2}$ & & 9539410 & 9581549 & 9651364 & 9527027 \\
\hline 33 & $1 s^{2} 2 s^{2} 2 p 3 p$ & ${ }^{1} D_{2}$ & & 9618893 & 9616237 & 9726710 & 9601264 \\
\hline 34 & $1 s^{2} 2 s^{2} 2 p 3 p$ & ${ }^{1} S_{0}$ & & 9665454 & 9653537 & 9782742 & 9660503 \\
\hline 35 & $1 s^{2} 2 s^{2} 2 p 3 d$ & ${ }^{1} D_{2}$ & & 9784684 & 9663951 & 9892314 & \\
\hline 36 & $1 s^{2} 2 s^{2} 2 p 3 d$ & ${ }^{3} F_{2}$ & & 9645548 & 9669602 & 9709788 & \\
\hline 37 & $1 s^{2} 2 s^{2} 2 p 3 d$ & ${ }^{3} F_{3}$ & & 9642585 & 9686381 & 9757732 & \\
\hline 38 & $1 s^{2} 2 s^{2} 2 p 3 d$ & ${ }^{3} F_{4}$ & & 9770912 & 9722518 & 9888034 & \\
\hline 39 & $1 s^{2} 2 s^{2} 2 p 3 d$ & ${ }^{3} D_{1}$ & & 9819196 & 9790906 & 9778582 & \\
\hline 40 & $1 s^{2} 2 s^{2} 2 p 3 d$ & ${ }^{3} D_{3}$ & & 9774819 & 9797787 & 9934750 & \\
\hline 41 & $1 s^{2} 2 s^{2} 2 p 3 d$ & ${ }^{3} D_{2}$ & & 9799674 & 9821907 & 9917312 & \\
\hline 42 & $1 s^{2} 2 s^{2} 2 p 3 d$ & ${ }^{3} P_{2}$ & & 9816420 & 9839432 & 9960351 & \\
\hline 43 & $1 s^{2} 2 s^{2} 2 p 3 d$ & ${ }^{3} P_{1}$ & & 9879365 & 9839520 & 9890218 & \\
\hline 44 & $1 s^{2} 2 s^{2} 2 p 3 d$ & ${ }^{3} P_{0}$ & & 9819196 & 9842066 & 9832815 & \\
\hline 45 & $1 s^{2} 2 s^{2} 2 p 3 d$ & ${ }^{1} F_{3}$ & & 9864046 & 9900369 & 9994644 & \\
\hline 46 & $1 s^{2} 2 s^{2} 2 p 3 d$ & ${ }^{1} P_{1}$ & & 9960132 & 9904890 & 9999604 & \\
\hline
\end{tabular}

${ }^{a}$ G. Tachiev, method: MCHF (ab initio) (2004);

${ }^{b}$ C. Froese Fischer, method: MCDHF (SD, cv, ab initio), Sept. (2009) 
TABLE II

Transition probabilities $\left(A\right.$ in $\left.\mathrm{s}^{-1}\right)$ and oscillator strengths $(g f)$ for carbon-like Ni XXIII $\left(a \pm b=a \times 10^{ \pm b}\right)$.

\begin{tabular}{|c|c|c|c|c|c|c|}
\hline \multirow{2}{*}{ Transition } & \multicolumn{2}{|c|}{ Present } & \multicolumn{2}{|c|}{$\mathrm{MCHF}$} & \multicolumn{2}{|c|}{ MCDHF } \\
\hline & $\mathrm{A}$ & gf & $\mathrm{A}$ & gf & $\mathrm{A}$ & gf \\
\hline \multicolumn{7}{|c|}{$1 s^{2} 2 s^{2} 2 p^{2}-1 s^{2} 2 s 2 p^{3}$} \\
\hline${ }^{3} P_{2}-{ }^{5} S_{2}$ & $6.85(+7)$ & $2.45(-3)$ & $7.27(+7)$ & $3.10(-3)$ & $6.28(+7)$ & $2.62(-3)$ \\
\hline${ }^{3} P_{1}-{ }^{5} S_{2}$ & $8.98(+7)$ & $2.74(-3)$ & $9.21(+7)$ & $2.86(-3)$ & $8.30(+7)$ & $2.74(-3)$ \\
\hline${ }^{3} P_{0}-{ }^{3} D_{1}$ & $1.63(+10)$ & $9.05(-2)$ & $2.02(+10)$ & $9.91(-2)$ & $1.63(+10)$ & $9.15(-2)$ \\
\hline${ }^{3} P_{0}-{ }^{3} P_{1}$ & $4.54(+9)$ & $1.72(-2)$ & $5.15(+9)$ & $1.49(-2)$ & $4.53(+9)$ & $1.75(-2)$ \\
\hline${ }^{3} P_{0^{-}}{ }^{3} S_{1}$ & $1.08(+10)$ & $2.86(-2)$ & $1.17(+10)$ & $3.03(-2)$ & $1.04(+10)$ & $2.67(-2)$ \\
\hline${ }^{3} P_{0}-{ }^{1} P_{1}$ & $3.72(+7)$ & $7.22(-5)$ & $4.06(+7)$ & $7.78(-5)$ & $3.67(+7)$ & $7.70(-5)$ \\
\hline${ }^{3} P_{1}-{ }^{3} D_{1}$ & $4.46(+8)$ & $3.83(-3)$ & $5.02(+8)$ & $3.20(-3)$ & $4.67(+8)$ & $3.40(-3)$ \\
\hline${ }^{3} P_{1}-{ }^{3} D_{2}$ & $1.18(+10)$ & $1.32(-1)$ & $1.37(+10)$ & $1.43(-1)$ & $1.12(+10)$ & $1.34(-1)$ \\
\hline${ }^{3} P_{1}-{ }^{3} P_{0}$ & $2.79(+10)$ & $4.32(-2)$ & $3.21(+10)$ & $4.69(-2)$ & $2.72(+10)$ & $4.46(-2)$ \\
\hline${ }^{3} P_{1}-{ }^{3} P_{1}$ & $2.37(+10)$ & $9.98(-2)$ & $2.75(+10)$ & $1.17(-1)$ & $2.29(+10)$ & $1.09(-1)$ \\
\hline${ }^{3} P_{1}-{ }^{3} P_{2}$ & $1.31(+8)$ & $9.76(-4)$ & $9.31(+7)$ & $6.27(-4)$ & $1.29(+8)$ & $9.76(-4)$ \\
\hline${ }^{3} P_{1}-{ }^{3} S_{1}$ & $2.74(+10)$ & $9.22(-2)$ & $3.06(+10)$ & $9.63(-2)$ & $2.71(+10)$ & $9.37(-2)$ \\
\hline${ }^{3} P_{1}-{ }^{1} D_{2}$ & $6.35(+8)$ & $3.00(-3)$ & $7.37(+8)$ & $3.50(-3)$ & $6.18(+8)$ & $3.22(-3)$ \\
\hline${ }^{3} P_{1}-{ }^{1} P_{1}$ & $7.15(+9)$ & $1.71(-2)$ & $7.77(+9)$ & $1.75(-2)$ & $6.79(+9)$ & $1.66(-2)$ \\
\hline${ }^{3} P_{2}-{ }^{3} D_{1}$ & $2.13(+8)$ & $1.97(-2)$ & $2.99(+8)$ & $2.18(-3)$ & $2.16(+8)$ & $1.80(-2)$ \\
\hline${ }^{3} P_{2}-{ }^{3} D_{2}$ & $9.27(+7)$ & $1.08(-3)$ & $1.19(+8)$ & $1.42(-3)$ & $7.08(+7)$ & $9.72(-4)$ \\
\hline${ }^{3} P_{2}-{ }^{3} D_{3}$ & $7.58(+9)$ & $1.02(-1)$ & $8.62(+9)$ & $1.28(-1)$ & $6.97(+9)$ & $1.20(-1)$ \\
\hline${ }^{3} P_{2}-{ }^{3} P_{1}$ & $2.81(+9)$ & $9.03(-2)$ & $2.67(+9)$ & $1.26(-2)$ & $2.35(+9)$ & $1.26(-2)$ \\
\hline${ }^{3} P_{2}-{ }^{3} P_{2}$ & $2.78(+10)$ & $2.27(-1)$ & $3.27(+10)$ & $2.44(-1)$ & $2.73(+10)$ & $2.29(-1)$ \\
\hline${ }^{3} P_{2}-{ }^{3} S_{1}$ & $7.49(+10)$ & $2.52(-1)$ & $8.31(+10)$ & $2.85(-1)$ & $7.22(+10)$ & $2.73(-1)$ \\
\hline${ }^{3} P_{2}-{ }^{1} D_{2}$ & $1.11(+10)$ & $6.25(-2)$ & $1.26(+10)$ & $6.54(-2)$ & $1.11(+10)$ & $6.34(-2)$ \\
\hline${ }^{3} P_{2}-{ }^{1} P_{1}$ & $4.79(+8)$ & $1.14(-3)$ & $4.32(+8)$ & $1.05(-3)$ & $4.91(+8)$ & $1.30(-3)$ \\
\hline${ }^{1} D_{2}-{ }^{3} D_{1}$ & $2.71(+8)$ & $3.42(-3)$ & $3.37(+8)$ & $3.94(-3)$ & $2.51(+8)$ & $3.49(-3)$ \\
\hline${ }^{1} D_{2}-{ }^{3} D_{2}$ & $4.63(+7)$ & $1.01(-3)$ & $5.86(+7)$ & $1.14(-3)$ & $4.23(+7)$ & $9.57(-4)$ \\
\hline${ }^{1} D_{2}-{ }^{3} D_{3}$ & $1.63(+9)$ & $4.02(-2)$ & $1.94(+9)$ & $4.51(-2)$ & $1.46(+9)$ & $4.02(-2)$ \\
\hline${ }^{1} D_{2}-{ }^{3} P_{1}$ & $1.98(+8)$ & $1.56(-3)$ & $2.16(+8)$ & $1.49(-3)$ & $2.03(+8)$ & $1.61(-3)$ \\
\hline${ }^{1} D_{2}-{ }^{3} P_{2}$ & $9.94(+7)$ & $2.07(-3)$ & $8.77(+7)$ & $9.41(-4)$ & $9.21(+7)$ & $1.31(-3)$ \\
\hline${ }^{1} D_{2}-{ }^{3} S_{1}$ & $8.13(+8)$ & $4.09(-3)$ & $8.84(+8)$ & $4.16(-3)$ & $7.77(+7)$ & $4.05(-3)$ \\
\hline${ }^{1} D_{2}-{ }^{1} D_{2}$ & $5.45(+10)$ & $3.12(-1)$ & $5.92(+10)$ & $4.12(-1)$ & $5.03(+10)$ & $3.90(-1)$ \\
\hline${ }^{1} D_{2}-{ }^{1} P_{1}$ & $7.79(+10)$ & $2.28(-1)$ & $9.01(+10)$ & $2.84(-1)$ & $7.84(+10)$ & $2.72(-1)$ \\
\hline${ }^{1} S_{0^{-}}{ }^{3} D_{1}$ & $5.97(+7)$ & $1.03(-3)$ & $7.44(+7)$ & $1.44(-3)$ & $5.18(+7)$ & $1.26(-3)$ \\
\hline${ }^{1} S_{0^{-}}{ }^{3} P_{1}$ & $2.66(+8)$ & $2.20(-3)$ & $2.86(+8)$ & $2.88(-3)$ & $2.15(+8)$ & $2.56(-3)$ \\
\hline${ }^{1} S_{0^{-}}{ }^{3} S_{1}$ & $1.02(+9)$ & $7.28(-3)$ & $1.25(+8)$ & $8.00(-3)$ & $9.87(+8)$ & $7.14(-3)$ \\
\hline${ }^{1} S_{0^{-}}{ }^{1} P_{1}$ & $2.09(+10)$ & $9.24(-2)$ & $2.41(+10)$ & $9.73(-2)$ & $2.04(+10)$ & $9.21(-2)$ \\
\hline \multicolumn{7}{|c|}{$1 s^{2} 2 s^{2} 2 p^{2}-1 s^{2} 2 s 2 p 3 s$} \\
\hline${ }^{3} P_{0^{-}}{ }^{3} P_{1}$ & $1.01(+12)$ & $5.12(-2)$ & $9.99(+11)$ & $5.29(-2)$ & $9.90(+11)$ & $5.35(-2)$ \\
\hline${ }^{3} P_{0^{-}}{ }^{1} P_{1}$ & $1.71(+10)$ & $8.15(-4)$ & $1.53(+10)$ & $7.81(-4)$ & $1.68(+10)$ & $8.72(-4)$ \\
\hline${ }^{3} P_{1}-{ }^{3} P_{0}$ & $3.04(+12)$ & $5.23(-2)$ & $2.82(+12)$ & $5.12(-2)$ & $2.91(+12)$ & $5.37(-2)$ \\
\hline${ }^{3} P_{1}-{ }^{3} P_{1}$ & $5.33(+11)$ & $3.05(-2)$ & $5.14(+11)$ & $2.79(-2)$ & $5.27(+11)$ & $2.92(-2)$ \\
\hline${ }^{3} P_{1}-{ }^{3} P_{2}$ & $8.48(+11)$ & $7.55(-2)$ & $8.57(+11)$ & $7.50(-2)$ & $8.48(+11)$ & $7.56(-2)$ \\
\hline${ }^{3} P_{1}-{ }^{1} P_{1}$ & $1.85(+11)$ & $1.06(-3)$ & $1.96(+11)$ & $1.02(-3)$ & $1.81(+11)$ & $9.63(-3)$ \\
\hline${ }^{3} P_{2}-{ }^{3} P_{1}$ & $1.89(+12)$ & $1.00(-1)$ & $1.81(+12)$ & $9.93(-2)$ & $1.85(+12)$ & $1.04(-1)$ \\
\hline${ }^{3} P_{2}-{ }^{3} P_{2}$ & $1.73(+12)$ & $1.36(-1)$ & $1.64(+12)$ & $1.45(-1)$ & $1.61(+12)$ & $1.45(-1)$ \\
\hline${ }^{3} P_{2}-{ }^{1} P_{1}$ & $2.89(+11)$ & $1.08(-2)$ & $2.65(+11)$ & $1.40(-1)$ & $2.29(+11)$ & $1.23(-2)$ \\
\hline${ }^{1} D_{2}-{ }^{3} P_{1}$ & $9.01(+10)$ & $5.21(-3)$ & $8.99(+10)$ & $5.13(-3)$ & $8.93(+10)$ & $5.19(-3)$ \\
\hline
\end{tabular}


TABLE II (cont.)

\begin{tabular}{|c|c|c|c|c|c|c|}
\hline \multirow{2}{*}{ Transition } & \multicolumn{2}{|c|}{ Present } & \multicolumn{2}{|c|}{$\mathrm{MCHF}$} & \multicolumn{2}{|c|}{ MCDHF } \\
\hline & $\mathrm{A}$ & gf & $\mathrm{A}$ & gf & $\mathrm{A}$ & gf \\
\hline${ }^{1} D_{2-}{ }^{3} P_{2}$ & $7.34(+11)$ & $6.51(-2)$ & $7.20(+11)$ & $6.61(-2)$ & $7.09(+11)$ & $6.64(-2)$ \\
\hline${ }^{1} D_{2}-{ }^{1} P_{1}$ & $3.12(+12)$ & $1.74(-1)$ & $3.21(+12)$ & $1.76(-1)$ & $3.09(+12)$ & $1.72(-1)$ \\
\hline${ }^{1} S_{0^{-}}{ }^{3} P_{1}$ & $3.91(+10)$ & $2.07(-3)$ & $3.78(+10)$ & $2.23(-3)$ & $3.65(+10)$ & $2.19(-3)$ \\
\hline${ }^{1} S_{0^{-}}{ }^{1} P_{1}$ & $1.33(+12)$ & $6.02(-2)$ & $1.13(+12)$ & $6.39(-2)$ & $1.09(+12)$ & $6.33(-2)$ \\
\hline \multicolumn{7}{|c|}{$1 s^{2} 2 s 2 p^{3}-1 s^{2} 2 p^{4}$} \\
\hline${ }^{5} S_{2}-{ }^{3} P_{2}$ & $2.86(+9)$ & $1.21(-2)$ & $3.09(+9)$ & $1.34(-2)$ & $2.78(+9)$ & $1.27(-2)$ \\
\hline${ }^{5} S_{2-}{ }^{3} P_{1}$ & $3.11(+8)$ & $6.42(-4)$ & $3.00(+8)$ & $6.38(-4)$ & $3.21(+8)$ & $7.22(-4)$ \\
\hline${ }^{5} S_{2}-^{1} D_{2}$ & $1.19(+9)$ & $3.91(-4)$ & $1.24(+8)$ & $3.94(-4)$ & $1.15(+8)$ & $3.86(-4)$ \\
\hline${ }^{3} D_{1}-{ }^{3} P_{2}$ & $5.86(+9)$ & $4.13(-2)$ & $6.31(+9)$ & $4.67(-2)$ & $5.53(+9)$ & $4.38(-2)$ \\
\hline${ }^{3} D_{1-}{ }^{3} P_{1}$ & $1.68(+10)$ & $6.03(-2)$ & $1.76(+10)$ & $6.04(-2)$ & $1.64(+10)$ & $6.04(-2)$ \\
\hline${ }^{3} D_{1}-{ }^{3} P_{0}$ & $4.39(+10)$ & $5.14(-2)$ & $4.47(+10)$ & $5.37(-2)$ & $4.12(+10)$ & $5.25(-2)$ \\
\hline${ }^{3} D_{1}-{ }^{1} S_{0}$ & $2.91(+8)$ & $1.98(-4)$ & $3.18(+8)$ & $2.13(-4)$ & $2.96(+8)$ & $2.09(-4)$ \\
\hline${ }^{3} D_{2}-{ }^{3} P_{2}$ & $1.72(+10)$ & $1.12(-1)$ & $1.82(+10)$ & $1.37(-1)$ & $1.64(+10)$ & $1.31(-1)$ \\
\hline${ }^{3} D_{2}-{ }^{3} P_{1}$ & $2.37(+10)$ & $8.55(-2)$ & $2.53(+10)$ & $8.78(-2)$ & $2.37(+10)$ & $8.83(-2)$ \\
\hline${ }^{3} D_{2}-{ }^{1} D_{2}$ & $9.54(+8)$ & $5.62(-3)$ & $1.01(+9)$ & $5.08(-3)$ & $8.87(+8)$ & $5.29(-3)$ \\
\hline${ }^{3} D_{3}-{ }^{3} P_{2}$ & $3.44(+10)$ & $2.66(-1)$ & $3.34(+10)$ & $2.75(-1)$ & $3.07(+10)$ & $2.70(-1)$ \\
\hline${ }^{3} D_{3}-{ }^{1} D_{2}$ & $8.41(+9)$ & $4.57(-2)$ & $9.46(+9)$ & $5.13(-2)$ & $8.50(+9)$ & $4.89(-2)$ \\
\hline${ }^{3} P_{0}-{ }^{3} P_{1}$ & $6.43(+9)$ & $2.99(-2)$ & $6.74(+9)$ & $3.20(-2)$ & $6.05(+9)$ & $3.11(-2)$ \\
\hline${ }^{3} P_{1}-{ }^{3} P_{0}$ & $2.35(+10)$ & $3.48(-2)$ & $2.24(+10)$ & $3.88(-2)$ & $2.04(+10)$ & $3.79(-2)$ \\
\hline${ }^{3} P_{1}-{ }^{3} P_{1}$ & $7.96(+8)$ & $3.46(-3)$ & $9.78(+8)$ & $4.77(-3)$ & $7.62(+8)$ & $4.04(-3)$ \\
\hline${ }^{3} P_{1}-{ }^{3} P_{2}$ & $4.41(+9)$ & $5.11(-2)$ & $4.84(+9)$ & $5.40(-2)$ & $4.29(+9)$ & $5.19(-2)$ \\
\hline${ }^{3} P_{1}-{ }^{1} D_{2}$ & $2.32(+9)$ & $1.41(-2)$ & $2.48(+9)$ & $1.72(-2)$ & $2.20(+9)$ & $1.63(-2)$ \\
\hline${ }^{3} P_{1}-{ }^{1} S_{0}$ & $7.13(+9)$ & $6.01(-3)$ & $7.97(+9)$ & $6.98(-3)$ & $7.02(+9)$ & $6.49(-3)$ \\
\hline${ }^{3} P_{2}-{ }^{3} P_{2}$ & $3.91(+9)$ & $5.17(-2)$ & $4.42(+9)$ & $5.29(-2)$ & $3.96(+9)$ & $5.13(-2)$ \\
\hline${ }^{3} P_{2}-{ }^{3} P_{1}$ & $2.01(+10)$ & $1.15(-1)$ & $2.28(+10)$ & $1.18(-1)$ & $2.00(+10)$ & $1.12(-1)$ \\
\hline${ }^{3} P_{2}-{ }^{1} D_{2}$ & $5.78(+9)$ & $4.14(-2)$ & $6.57(+9)$ & $4.80(-2)$ & $5.66(+9)$ & $4.44(-2)$ \\
\hline${ }^{3} S_{1}-{ }^{3} P_{2}$ & $7.17(+9)$ & $1.09(-1)$ & $7.46(+9)$ & $1.33(-1)$ & $6.43(+9)$ & $1.28(-1)$ \\
\hline${ }^{3} S_{1}-{ }^{3} P_{1}$ & $1.80(+10)$ & $1.39(-1)$ & $1.97(+10)$ & $1.43(-1)$ & $1.69(+10)$ & $1.36(-1)$ \\
\hline${ }^{3} S_{1}-{ }^{3} P_{0}$ & $2.67(+10)$ & $6.07(-2)$ & $2.62(+10)$ & $6.81(-2)$ & $2.28(+10)$ & $6.51(-2)$ \\
\hline${ }^{3} S_{1}-{ }^{1} D_{2}$ & $3.69(+7)$ & $3.59(-4)$ & $4.22(+7)$ & $4.18(-4)$ & $3.08(+7)$ & $3.36(-4)$ \\
\hline${ }^{3} S_{1}-{ }^{1} S_{0}$ & $6.09(+9)$ & $7.01(-3)$ & $6.42(+9)$ & $7.43(-3)$ & $6.05(+9)$ & $7.50(-3)$ \\
\hline${ }^{1} D_{2}-{ }^{3} P_{2}$ & $9.06(+8)$ & $2.76(-2)$ & $1.02(+9)$ & $2.20(-2)$ & $8.75(+8)$ & $2.10(-2)$ \\
\hline${ }^{1} D_{2}-{ }^{3} P_{1}$ & $8.32(+8)$ & $8.55(-3)$ & $1.06(+9)$ & $9.01(-3)$ & $8.49(+8)$ & $7.94(-3)$ \\
\hline${ }^{1} D_{2}-{ }^{1} D_{2}$ & $3.61(+10)$ & $4.34(-1)$ & $4.08(+10)$ & $4.64(-1)$ & $3.61(+10)$ & $4.33(-1)$ \\
\hline${ }^{1} P_{1}-{ }^{3} P_{2}$ & $1.08(+8)$ & $4.15(-3)$ & $1.17(+8)$ & $4.65(-3)$ & $9.36(+7)$ & $4.33(-3)$ \\
\hline${ }^{1} P_{1}-{ }^{3} P_{1}$ & $1.01(+9)$ & $1.32(-2)$ & $1.15(+9)$ & $1.58(-2)$ & $9.26(+8)$ & $1.44(-2)$ \\
\hline${ }^{1} P_{1}-{ }^{3} P_{0}$ & $4.17(+7)$ & $2.04(-4)$ & $5.64(+7)$ & $2.86(-4)$ & $4.02(+7)$ & $2.27(-4)$ \\
\hline${ }^{1} P_{1}-{ }^{1} D_{2}$ & $5.71(+9)$ & $9.98(-2)$ & $6.29(+9)$ & $1.11(-1)$ & $5.31(+9)$ & $1.04(-1)$ \\
\hline${ }^{1} P_{1}-{ }^{1} S_{0}$ & $9.10(+10)$ & $1.59(-1)$ & $1.00(+11)$ & $1.76(-1)$ & $9.05(+10)$ & $1.72(-1)$ \\
\hline \multicolumn{7}{|c|}{$1 s^{2} 2 s^{2} 2 p^{2}-1 s^{2} 2 s^{2} 2 p 3 p$} \\
\hline${ }^{5} S_{2-}{ }^{1} P_{1}$ & $2.60(+10)$ & $1.19(-3)$ & $2.47(+10)$ & $1.42(-3)$ & $2.27(+10)$ & $1.32(-3)$ \\
\hline${ }^{5} S_{2}-^{3} D_{1}$ & $2.97(+8)$ & $2.08(-5)$ & $3.46(+8)$ & $2.02(-5)$ & $3.05(+8)$ & $1.80(-5)$ \\
\hline${ }^{5} S_{2}-{ }^{3} D_{2}$ & $1.70(+9)$ & $1.57(-4)$ & $1.61(+9)$ & $1.54(-4)$ & $1.60(+9)$ & $1.55(-4)$ \\
\hline${ }^{5} S_{2}{ }^{-3} D_{3}$ & $4.98(+8)$ & $6.92(-5)$ & $3.31(+8)$ & $4.31(-5)$ & $4.10(+8)$ & $5.39(-5)$ \\
\hline${ }^{5} S_{2}-{ }^{3} P_{1}$ & $2.34(+10)$ & $1.02(-3)$ & $1.99(+10)$ & $1.15(-3)$ & $2.12(+10)$ & $1.20(-3)$ \\
\hline${ }^{5} S_{2}-{ }^{3} P_{2}$ & $4.33(+9)$ & $3.68(-4)$ & $3.26(+9)$ & $3.01(-4)$ & $4.13(+9)$ & $3.88(-4)$ \\
\hline${ }^{5} S_{2}-{ }^{3} S_{1}$ & $2.99(+10)$ & $1.91(-3)$ & $3.41(+10)$ & $1.89(-3)$ & $2.98(+10)$ & $1.68(-3)$ \\
\hline${ }^{5} S_{2}-{ }^{1} D_{2}$ & $1.21(+10)$ & $1.02(-3)$ & $1.04(+10)$ & $9.45(-4)$ & $1.18(+10)$ & $1.09(-3)$ \\
\hline
\end{tabular}


TABLE II (cont.)

\begin{tabular}{|c|c|c|c|c|c|c|}
\hline \multirow{2}{*}{ Transition } & \multicolumn{2}{|c|}{ Present } & \multicolumn{2}{|c|}{$\mathrm{MCHF}$} & \multicolumn{2}{|c|}{ MCDHF } \\
\hline & $\mathrm{A}$ & gf & $\mathrm{A}$ & gf & $\mathrm{A}$ & gf \\
\hline \multicolumn{7}{|c|}{$1 s^{2} 2 s^{2} 2 p 3 s-1 s^{2} 2 s^{2} 2 p 3 p$} \\
\hline${ }^{3} P_{0}-{ }^{3} D_{1}$ & $3.69(+8)$ & $4.71(-2)$ & $5.05(+8)$ & $4.68(-2)$ & $3.61(+8)$ & $4.17(-2)$ \\
\hline${ }^{3} P_{0}-{ }^{1} P_{1}$ & $2.17(+9)$ & $1.05(-1)$ & $2.62(+9)$ & $1.36(-1)$ & $2.01(+9)$ & $1.24(-1)$ \\
\hline${ }^{3} P_{0}-{ }^{3} P_{1}$ & $1.37(+8)$ & $3.95(-3)$ & $1.66(+8)$ & $4.30(-3)$ & $1.43(+8)$ & $4.29(-3)$ \\
\hline${ }^{3} P_{1}-{ }^{3} D_{1}$ & $6.98(+8)$ & $9.12(-2)$ & $9.44(+8)$ & $9.57(-2)$ & $6.60(+8)$ & $8.45(-2)$ \\
\hline${ }^{3} P_{1}-{ }^{1} P_{1}$ & $6.88(+8)$ & $4.40(-2)$ & $8.84(+8)$ & $4.91(-2)$ & $6.65(+8)$ & $4.43(-2)$ \\
\hline${ }^{3} P_{1}-{ }^{3} D_{2}$ & $2.91(+9)$ & $2.86(-1)$ & $3.67(+9)$ & $3.26(-1)$ & $2.77(+9)$ & $2.96(-1)$ \\
\hline${ }^{3} P_{1}-{ }^{3} P_{0}$ & $3.29(+9)$ & $6.68(-2)$ & $4.18(+9)$ & $7.21(-2)$ & $3.25(+9)$ & $6.62(-2)$ \\
\hline${ }^{3} P_{1}-{ }^{3} P_{1}$ & $3.37(+8)$ & $1.55(-2)$ & $4.40(+8)$ & $1.19(-2)$ & $3.87(+8)$ & $1.22(-2)$ \\
\hline${ }^{3} P_{1}-{ }^{3} P_{2}$ & $5.11(+8)$ & $2.65(-2)$ & $6.92(+8)$ & $2.79(-2)$ & $5.71(+8)$ & $2.64(-2)$ \\
\hline${ }^{3} P_{1}-{ }^{3} S_{1}$ & $5.98(+7)$ & $1.82(-3)$ & $8.65(+7)$ & $2.05(-3)$ & $6.66(+7)$ & $1.91(-3)$ \\
\hline${ }^{3} P_{1}-{ }^{1} D_{2}$ & $2.57(+8)$ & $8.53(-3)$ & $3.45(+8)$ & $1.07(-2)$ & $2.82(+8)$ & $9.29(-3)$ \\
\hline${ }^{3} P_{1}-{ }^{1} S_{0}$ & $1.21(+9)$ & $6.95(-3)$ & $1.50(+9)$ & $7.10(-3)$ & $1.35(+9)$ & $7.08(-3)$ \\
\hline${ }^{3} P_{2}-{ }^{3} P_{1}$ & $5.51(+8)$ & $4.25(-2)$ & $7.37(+8)$ & $5.34(-2)$ & $5.18(+8)$ & $4.55(-2)$ \\
\hline${ }^{3} P_{2}-{ }^{3} D_{3}$ & $2.62(+9)$ & $2.95(-1)$ & $2.97(+9)$ & $4.28(-1)$ & $2.77(+9)$ & $2.96(-1)$ \\
\hline${ }^{3} P_{2}-{ }^{3} P_{2}$ & $2.22(+9)$ & $2.51(-2)$ & $2.53(+9)$ & $2.54(-1)$ & $1.97(+9)$ & $2.35(-1)$ \\
\hline${ }^{3} P_{2}-{ }^{3} S_{1}$ & $1.59(+9)$ & $1.38(-1)$ & $2.16(+9)$ & $1.26(-1)$ & $1.69(+9)$ & $1.18(-1)$ \\
\hline${ }^{3} P_{2}-{ }^{1} D_{2}$ & $8.12(+8)$ & $5.58(-2)$ & $1.06(+9)$ & $6.56(-2)$ & $8.18(+8)$ & $5.81(-2)$ \\
\hline${ }^{1} P_{1}-{ }^{3} P_{1}$ & $7.85(+8)$ & $8.92(-2)$ & $1.05(+9)$ & $9.41(-2)$ & $7.72(+8)$ & $8.62(-2)$ \\
\hline${ }^{1} P_{1}-{ }^{3} P_{2}$ & $3.45(+8)$ & $4.05(-2)$ & $4.03(+8)$ & $4.91(-2)$ & $2.81(+8)$ & $4.16(-2)$ \\
\hline${ }^{1} P_{1}-{ }^{3} S_{1}$ & $5.56(+8)$ & $4.42(-2)$ & $6.66(+8)$ & $4.74(-2)$ & $4.61(+8)$ & $4.00(-2)$ \\
\hline${ }^{1} P_{1}-{ }^{1} D_{2}$ & $3.96(+9)$ & $3.36(-1)$ & $4.66(+9)$ & $3.34(-1)$ & $3.69(+9)$ & $3.09(-1)$ \\
\hline${ }^{1} P_{1}-{ }^{1} S_{0}$ & $5.12(+9)$ & $5.61(-2)$ & $5.44(+9)$ & $5.66(-2)$ & $4.71(+9)$ & $5.49(-2)$ \\
\hline \multicolumn{7}{|c|}{$1 s^{2} 2 s 2 p^{3}-1 s^{2} 2 s^{2} 2 p 3 p$} \\
\hline${ }^{3} D_{1}-{ }^{3} D_{1}$ & $1.36(+10)$ & $8.52(-4)$ & $1.49(+10)$ & $9.38(-4)$ & $1.27(+10)$ & $8.06(-4)$ \\
\hline${ }^{3} D_{1}-{ }^{1} P_{1}$ & $9.91(+9)$ & $5.72(-4)$ & $8.86(+9)$ & $5.46(-4)$ & $8.25(+9)$ & $5.15(-4)$ \\
\hline${ }^{3} D_{1}-{ }^{3} D_{2}$ & $2.01(+9)$ & $2.68(-4)$ & $1.17(+9)$ & $1.21(-4)$ & $2.24(+9)$ & $2.33(-4)$ \\
\hline${ }^{3} D_{1}-{ }^{3} P_{0}$ & $3.19(+11)$ & $7.54(-3)$ & $3.17(+11)$ & $6.50(-3)$ & $3.12(+11)$ & $6.48(-3)$ \\
\hline${ }^{3} D_{1}-{ }^{3} P_{1}$ & $6.58(+10)$ & $3.02(-3)$ & $6.03(+10)$ & $3.61(-3)$ & $5.83(+10)$ & $3.54(-3)$ \\
\hline${ }^{3} D_{1}-{ }^{3} P_{2}$ & $2.32(+8)$ & $2.35(-5)$ & $1.28(+8)$ & $1.27(-5)$ & $1.94(+8)$ & $1.96(-5)$ \\
\hline${ }^{3} D_{1-}{ }^{3} S_{1}$ & $2.15(+9)$ & $1.46(-4)$ & $3.29(+9)$ & $1.96(-4)$ & $2.09(+9)$ & $1.26(-4)$ \\
\hline${ }^{3} D_{1}-{ }^{1} S_{0}$ & $1.23(+12)$ & $2.14(-2)$ & $1.24(+12)$ & $2.39(-2)$ & $1.16(+12)$ & $2.27(-2)$ \\
\hline${ }^{3} D_{2}-{ }^{3} D_{1}$ & $1.92(+8)$ & $1.04(-5)$ & $3.63(+8)$ & $2.28(-5)$ & $1.55(+8)$ & $9.86(-6)$ \\
\hline${ }^{3} D_{2}-{ }^{1} P_{1}$ & $1.71(+11)$ & $9.13(-3)$ & $1.49(+11)$ & $9.20(-3)$ & $1.35(+11)$ & $8.44(-3)$ \\
\hline${ }^{3} D_{2}-{ }^{3} D_{2}$ & $8.86(+9)$ & $9.92(-4)$ & $8.12(+9)$ & $8.34(-4)$ & $7.82(+9)$ & $8.21(-4)$ \\
\hline${ }^{3} D_{2}-{ }^{3} P_{1}$ & $1.12(+11)$ & $7.84(-3)$ & $1.20(+11)$ & $7.19(-3)$ & $1.24(+11)$ & $7.53(-3)$ \\
\hline${ }^{3} D_{2}-{ }^{3} D_{3}$ & $5.62(+9)$ & $8.49(-4)$ & $3.31(+9)$ & $4.62(-4)$ & $5.48(+9)$ & $7.74(-4)$ \\
\hline${ }^{3} D_{2}-{ }^{3} P_{2}$ & $2.72(+10)$ & $2.94(-3)$ & $2.58(+10)$ & $2.57(-3)$ & $2.59(+10)$ & $2.61(-3)$ \\
\hline${ }^{3} D_{2}-{ }^{3} S_{1}$ & $9.85(+9)$ & $5.79(-4)$ & $7.04(+9)$ & $4.20(-4)$ & $9.34(+9)$ & $5.64(-4)$ \\
\hline${ }^{3} D_{2}-{ }^{1} D_{2}$ & $6.15(+9)$ & $6.03(-4)$ & $5.85(+9)$ & $5.72(-4)$ & $5.76(+9)$ & $5.70(-4)$ \\
\hline${ }^{3} D_{3}-{ }^{3} D_{2}$ & $3.43(+10)$ & $3.69(-3)$ & $3.61(+10)$ & $3.75(-3)$ & $3.12(+10)$ & $3.27(-3)$ \\
\hline${ }^{3} D_{3}-{ }^{3} D_{3}$ & $1.98(+10)$ & $2.92(-3)$ & $1.93(+10)$ & $2.71(-3)$ & $1.82(+10)$ & $2.59(-3)$ \\
\hline${ }^{3} D_{3}-{ }^{3} P_{2}$ & $1.20(+11)$ & $1.50(-2)$ & $1.38(+11)$ & $1.39(-2)$ & $1.49(+11)$ & $1.52(-2)$ \\
\hline${ }^{3} D_{3}-{ }^{1} D_{2}$ & $1.16(+11)$ & $1.37(-2)$ & $1.37(+11)$ & $1.35(-2)$ & $1.21(+11)$ & $1.21(-2)$ \\
\hline${ }^{3} P_{0}-{ }^{3} D_{1}$ & $1.19(+10)$ & $7.88(-4)$ & $1.14(+10)$ & $7.49(-4)$ & $1.03(+10)$ & $6.81(-4)$ \\
\hline${ }^{3} P_{0}-{ }^{1} P_{1}$ & $5.73(+9)$ & $3.56(-4)$ & $5.31(+9)$ & $3.41(-4)$ & $4.84(+9)$ & $3.15(-4)$ \\
\hline${ }^{3} P_{0}-{ }^{3} P_{1}$ & $2.43(+10)$ & $1.75(-3)$ & $2.39(+10)$ & $1.50(-3)$ & $2.36(+10)$ & $1.50(-3)$ \\
\hline${ }^{3} P_{0}-{ }^{3} S_{1}$ & $2.19(+10)$ & $1.64(-3)$ & $2.22(+10)$ & $1.37(-3)$ & $2.08(+10)$ & $1.31(-3)$ \\
\hline${ }^{3} P_{1}-{ }^{3} D_{1}$ & $1.23(+10)$ & $7.42(-4)$ & $1.05(+10)$ & $6.72(-4)$ & $1.02(+10)$ & $6.80(-4)$ \\
\hline
\end{tabular}


TABLE II (cont.)

\begin{tabular}{|c|c|c|c|c|c|c|}
\hline \multirow{2}{*}{ Transition } & \multicolumn{2}{|c|}{ Present } & \multicolumn{2}{|c|}{$\mathrm{MCHF}$} & \multicolumn{2}{|c|}{ MCDHF } \\
\hline & $\mathrm{A}$ & gf & $\mathrm{A}$ & gf & $\mathrm{A}$ & gf \\
\hline${ }^{3} P_{1}-{ }^{3} D_{2}$ & $1.83(+10)$ & $2.04(-3)$ & $1.95(+10)$ & $2.10(-3)$ & $1.75(+10)$ & $1.90(-3)$ \\
\hline${ }^{3} P_{1}-{ }^{3} P_{0}$ & $1.41(+9)$ & $3.26(-5)$ & $2.35(+9)$ & $5.04(-5)$ & $1.26(+9)$ & $2.72(-5)$ \\
\hline${ }^{3} P_{1}-{ }^{3} P_{1}$ & $2.13(+10)$ & $1.01(-3)$ & $2.05(+10)$ & $1.26(-3)$ & $1.79(+10)$ & $1.14(-3)$ \\
\hline${ }^{3} P_{1}-{ }^{3} P_{2}$ & $1.98(+10)$ & $2.37(-3)$ & $2.02(+10)$ & $2.09(-3)$ & $1.89(+10)$ & $1.98(-3)$ \\
\hline${ }^{3} P_{1}-{ }^{3} S_{1}$ & $7.52(+10)$ & $4.39(-3)$ & $7.06(+10)$ & $4.39(-3)$ & $6.69(+10)$ & $4.22(-3)$ \\
\hline${ }^{3} P_{1}-{ }^{1} D_{2}$ & $3.73(+9)$ & $3.54(-4)$ & $3.55(+9)$ & $3.61(-4)$ & $3.44(+9)$ & $3.56(-4)$ \\
\hline${ }^{3} P_{1}-{ }^{1} S_{0}$ & $9.89(+10)$ & $2.58(-3)$ & $9.98(+10)$ & $1.98(-3)$ & $1.01(+11)$ & $2.06(-3)$ \\
\hline${ }^{3} P_{2}-{ }^{3} D_{1}$ & $3.99(+9)$ & $2.93(-4)$ & $5.73(+9)$ & $3.78(-4)$ & $3.29(+9)$ & $2.19(-4)$ \\
\hline${ }^{3} P_{2}-{ }^{1} P_{1}$ & $8.02(+9)$ & $5.12(-4)$ & $7.77(+9)$ & $5.04(-4)$ & $7.58(+9)$ & $4.97(-4)$ \\
\hline${ }^{3} P_{2}-{ }^{3} D_{2}$ & $1.75(+10)$ & $1.62(-3)$ & $1.54(+10)$ & $1.65(-3)$ & $1.50(+10)$ & $1.64(-3)$ \\
\hline${ }^{3} P_{2}-{ }^{3} P_{1}$ & $1.22(+10)$ & $8.55(-4)$ & $1.52(+10)$ & $9.59(-4)$ & $1.15(+10)$ & $7.51(-4)$ \\
\hline${ }^{3} P_{2}-{ }^{3} D_{3}$ & $3.89(+10)$ & $5.36(-3)$ & $3.52(+10)$ & $5.15(-3)$ & $3.41(+10)$ & $5.05(-3)$ \\
\hline${ }^{3} P_{2}-{ }^{3} P_{2}$ & $3.53(+10)$ & $3.95(-3)$ & $3.31(+10)$ & $3.46(-3)$ & $3.36(+10)$ & $3.55(-3)$ \\
\hline${ }^{3} P_{2}-{ }^{3} S_{1}$ & $4.98(+10)$ & $3.86(-3)$ & $4.94(+10)$ & $3.09(-3)$ & $4.53(+10)$ & $2.88(-3)$ \\
\hline${ }^{3} P_{2}-{ }^{1} D_{2}$ & $3.20(+9)$ & $2.73(-4)$ & $2.49(+9)$ & $2.56(-4)$ & $2.10(+9)$ & $2.18(-4)$ \\
\hline${ }^{3} S_{1}-{ }^{3} D_{1}$ & $6.32(+9)$ & $4.19(-4)$ & $6.13(+9)$ & $4.19(-4)$ & $5.88(+9)$ & $4.08(-4)$ \\
\hline${ }^{3} S_{1}-{ }^{1} P_{1}$ & $1.01(+10)$ & $7.48(-4)$ & $9.38(+9)$ & $6.30(-4)$ & $9.93(+9)$ & $6.75(-4)$ \\
\hline${ }^{3} S_{1}-{ }^{3} P_{0}$ & $1.54(+10)$ & $3.92(-4)$ & $1.33(+10)$ & $2.98(-4)$ & $1.40(+10)$ & $3.16(-4)$ \\
\hline${ }^{3} S_{1}-{ }^{3} P_{1}$ & $9.45(+8)$ & $6.13(-5)$ & $6.75(+8)$ & $4.39(-5)$ & $7.89(+8)$ & $5.22(-5)$ \\
\hline${ }^{3} S_{1}-{ }^{3} P_{2}$ & $8.62(+9)$ & $9.82(-4)$ & $7.26(+9)$ & $7.84(-4)$ & $8.32(+9)$ & $9.11(-4)$ \\
\hline${ }^{3} S_{1}-{ }^{3} S_{1}$ & $1.75(+9)$ & $1.49(-4)$ & $1.56(+9)$ & $1.01(-4)$ & $1.68(+9)$ & $1.11(-4)$ \\
\hline${ }^{3} S_{1}-{ }^{1} D_{2}$ & $2.01(+10)$ & $2.17(-3)$ & $1.99(+10)$ & $2.11(-3)$ & $1.97(+10)$ & $2.12(-3)$ \\
\hline${ }^{3} S_{1}-{ }^{1} S_{0}$ & $6.35(+10)$ & $1.51(-3)$ & $6.40(+10)$ & $1.34(-3)$ & $6.70(+10)$ & $1.42(-3)$ \\
\hline${ }^{1} D_{2}-{ }^{3} D_{1}$ & $5.21(+9)$ & $2.21(-4)$ & $5.35(+9)$ & $3.72(-4)$ & $4.81(+9)$ & $3.38(-4)$ \\
\hline${ }^{1} D_{2}-{ }^{3} D_{2}$ & $2.25(+9)$ & $3.12(-4)$ & $2.41(+9)$ & $2.73(-4)$ & $2.64(+9)$ & $3.03(-4)$ \\
\hline${ }^{1} D_{2}-{ }^{3} P_{1}$ & $6.12(+9)$ & $3.86(-4)$ & $6.06(+9)$ & $4.01(-4)$ & $8.59(+9)$ & $5.76(-4)$ \\
\hline${ }^{1} D_{2}-{ }^{3} D_{3}$ & $3.05(+9)$ & $5.12(-4)$ & $5.28(+9)$ & $8.11(-4)$ & $3.13(+9)$ & $4.87(-4)$ \\
\hline${ }^{1} D_{2}-{ }^{3} P_{2}$ & $7.27(+9)$ & $7.94(-4)$ & $6.50(+9)$ & $7.13(-4)$ & $6.95(+9)$ & $7.72(-4)$ \\
\hline${ }^{1} D_{2}-{ }^{3} S_{1}$ & $2.22(+10)$ & $1.19(-3)$ & $2.06(+10)$ & $1.35(-3)$ & $2.12(+10)$ & $1.41(-3)$ \\
\hline${ }^{1} D_{2}-{ }^{1} D_{2}$ & $8.02(+10)$ & $8.19(-3)$ & $7.09(+10)$ & $7.64(-3)$ & $8.17(+10)$ & $8.91(-3)$ \\
\hline${ }^{1} P_{1}-{ }^{3} D_{1}$ & $4.28(+9)$ & $3.02(-4)$ & $3.66(+9)$ & $2.64(-4)$ & $3.72(+9)$ & $2.72(-4)$ \\
\hline${ }^{1} P_{1}-{ }^{1} P_{1}$ & $1.12(+9)$ & $7.82(-5)$ & $1.03(+9)$ & $7.35(-5)$ & $1.09(+9)$ & $7.84(-5)$ \\
\hline${ }^{1} P_{1}-{ }^{3} D_{2}$ & $7.72(+8)$ & $8.07(-5)$ & $6.63(+8)$ & $7.82(-5)$ & $6.69(+8)$ & $7.99(-5)$ \\
\hline${ }^{1} P_{1}-{ }^{3} P_{0}$ & $4.69(+9)$ & $1.06(-4)$ & $4.23(+9)$ & $9.96(-5)$ & $4.58(+9)$ & $1.09(-4)$ \\
\hline${ }^{1} P_{1}-{ }^{3} P_{1}$ & $7.52(+8)$ & $6.23(-4)$ & $7.45(+9)$ & $5.12(-4)$ & $7.67(+9)$ & $5.34(-4)$ \\
\hline${ }^{1} P_{1}-{ }^{3} P_{2}$ & $1.12(+10)$ & $1.31(-3)$ & $1.10(+10)$ & $1.25(-3)$ & $1.06(+10)$ & $1.23(-3)$ \\
\hline${ }^{1} P_{1-}{ }^{3} S_{1}$ & $3.92(+9)$ & $2.97(-4)$ & $4.06(+9)$ & $2.77(-4)$ & $3.79(+9)$ & $2.62(-4)$ \\
\hline${ }^{1} P_{1}-{ }^{1} D_{2}$ & $3.60(+9)$ & $4.07(-4)$ & $3.92(+9)$ & $4.37(-4)$ & $3.71(+9)$ & $4.20(-4)$ \\
\hline${ }^{1} P_{1}-{ }^{1} S_{0}$ & $1.81(+11)$ & $3.59(-3)$ & $1.44(+11)$ & $3.19(-3)$ & $1.68(+11)$ & $3.76(-3)$ \\
\hline
\end{tabular}

